\title{
THE MAGNETOHYDRODYNAMIC DEVELOPMENT OF TWO-RIBBON FLARES OR A FIVE-FINGER THEORY FOR SOLAR FLARES
}

\author{
J. S. Kaastra \\ Sterrewacht Sonnenborgh, Zonnenburg 2, 3512 NL Utrecht, \\ The Netherlands
}

\begin{abstract}
A semi-analytical model for the electrodynamic development of two-ribbon flares is presented. A current filament above a bipolar active region starts rising according to the model of Van Tend and Kuperus. Due to this motion large induced electric fields arise at a magnetic neutral line far below the filament, resulting in and associated with magnetic reconnection and the formation of a current sheet. The interaction of this current sheet with the original current filament, the background magnetic field and the boundary layer of the photosphere determine the further electrodynamic development of the flare. The model predicts the energy release, the time of maximum, the height of the energy source and other quantities reasonably we11.
\end{abstract}

\section{SUMMARY}

The magnetic fields in a flare can be described by means of their sources, the currents. Here the total field is splitted into 5 components, which are treated below. 1. The background photospherical field $B$ has its source below the surface, is in general bipolar along a polarity reversal line. It does not change significantly during the flare, even if emergent flux triggers the flare. It can be obtained easily from magnetograms, and may be represented by a simple dipole or line dipole which is placed below the surface. 2. The filament may be represented by a current at height $h$ above the surface above the polarity reversal line, with radius small compared to its height and initial length large compared to its height. Any magnetic field with its source in the corona (like the filament) cannot penetrate into the surface on short time scales, because of the high conductivity and inertia of the photosphere. Therefore at the surface of the Sun these fields must be parallel to it. Formally this may be represented by placing a virtual mirror current of opposite direction below the surface $/ 1 /$. This virtual field represents the boundary condition. 3. The height of this mirror filament is determined by the condition of force balance on the filament before the flare, i.e. the filament is at such a height where apart from its own field a neutral line would exist. During the slow preflare energy build up phase the filament will come at a position where the background field falls off more rapidly then the mirrorfilament field, and no stable equilibrium is possible anymore /2/. Near this height any small disturbance like emergent flux or shocks from a flare (precursor) at another place may trigger the flare. The filament will rise without bound. From the analytic expression for the three field components it can be seen that a neutral line will appear at the surface as soon as the filament has started rising. This neutral line also rises, but stays well below the filament. From the Maxwell equation $\nabla \times E=-\partial B / \partial t$ and the given fields $B(t) E$ can be calculated explicitly. It is nonzero at the neutral line.

4. Therefore a current sheet will be formed at the neutral 1 ine /3/. At this sheet the reconnection takes place and the magnetic energy is dissipated. For the same reasons as the existence of the mirror filament also

5. a vitual mirror current sheet is needed. The height of the sheet and its current must be determined in such a way as to cause a neutral line at the position of the filament and the sheet, apart of course from the own fields of the respective two currents. Typical results are a sheet current $10 \%$ of the filament current (at the time of maximum) and a sheet height $30 \%$ of the height of the rising filament, and induced electric fields at the sheet of order $100 \mathrm{~V} / \mathrm{m}$.

It can be seen that the ribbon distance increases with time, the size of the current sheet increases and also the height of the sheet, to a height of the order of the initial filament height. Condensation of the current sheet might therefore in the later phase of the flare cause the re-appearance of a filament. 
The heat produced in the sheet and the fast particles which are accelerated there are transported downwards along the magnetic field to produce the optical flare ribbons, hard $X$-ray bursts and also $\mathrm{cm}$ radiation (note that the magnitude of the magnetic field increases from the current sheet (for example .002 T) to the footpoints (for example $.04 \mathrm{~T}$ ). Another part of the released energy will enter the closed reconnected loops around the rising filament, and will result into heating and associated X-ray arches, which are observed e.g. with HXIS at the SMM satelite.

This work was financed by the Dutch Organization for the Advancement of Pure Science (2.W.O.). The extended paper was submitted to Astron. and Astrophys.

\section{REFERENCES}

1. M. Kuperus and M.A. Raadu, The support of prominences formed in neutral sheets, Astron. Astrophys. 31,189 (1974)

2. W. van Tend and $M$. Kuperus, The development of coronal electric current systems in active regions and their relation to filaments and flares, Solar Physics 59, 115 (1978)

3. S.I. Syrovatskii, Formation of current sheets in a plasma with a frozen-in strong magnetic field, 2h. Eksp. Teor. Fiz. 60, 1727 (1971)
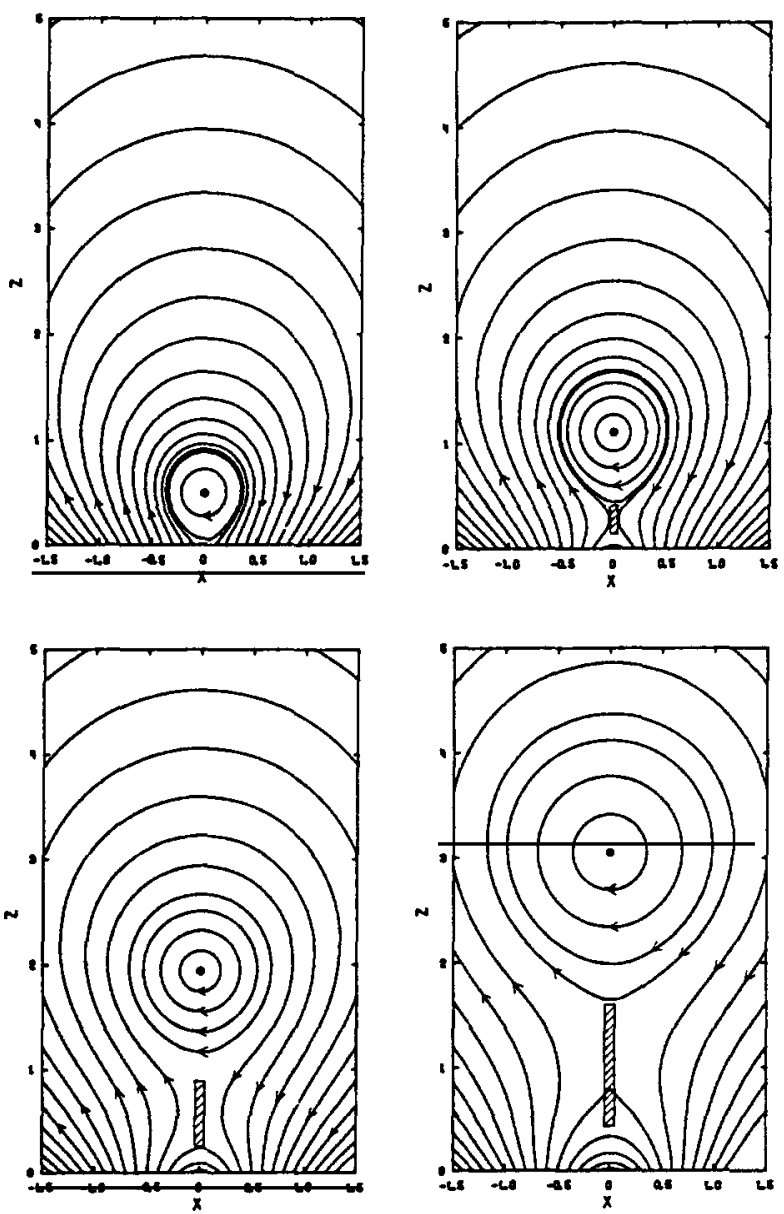

Fig. 1. Evolution of the flare magnetic field. a. $t=0.0, b, t=0.6, c, t=1.2, d, t=1.8$ unit of time is typically $1000 \mathrm{~s}$ for large flares, unit for distance $50000 \mathrm{~km}$.

The filament is indicated by the dot in the centre of the closed field lines and the current sheet by the dashed area at the $\mathrm{X}$-point. 